\title{
Motricidad, en-acción y fenomenología: la articulación conceptual de la existencia
}

\author{
Motricity, en-action and phenomenology: the conceptual articulation of existence \\ Motricidade, em-ação e fenomenologia: a articulação conceitual da existência \\ Sergio Alejandro Toro-Arévalo ${ }^{1}$ \\ Universidad Austral de Chile, UACh, Valdivia, Chile
}

\begin{abstract}
RESUMEN
El presente trabajo pretende hacer un análisis de vinculación entre la en-acción, la motricidad y la fenomenología con el propósito de establecer los vínculos y articulaciones tanto a nivel conceptual como programático de los campos de estudio nombrados. En este sentido, se ha concretado que desde la perspectiva de sus bases se encuentran en la misma raíz, y que sus posibilidades de encuentro son absolutamente complementarias. La fenomenología como punto de partida del enfoque en-activo de la motricidad crea las condiciones óptimas para la confluencia y reciprocidad de métodos y categorías de análisis de la problemática existencial de la actualidad. El núcleo o eje central del enfoque en-activo es constituido por los siguientes aspectos: autonomía, encarnación, experiencia, hacer-sentido y emergencia. Palabras clave: En-acción. Motricidad Humana. Fenomenología.
\end{abstract}

\begin{abstract}
The present work intends to analyze the relations between en-action, motricity and phenomenology aiming to establish links and articulations both conceptually and programmatically of the mentioned fields of study. In this sense, based in the analysis of their perspectives and foundations, it was found the same basis, indicating that they are absolutely complementary. Phenomenology as a starting point for the en-action approach to human motricity creates the ideal conditions for the confluence and reciprocity of methods and categories of analysis of today's existential problems. The nucleus or central axis of the enaction approach consists of the following aspects: autonomy, incarnation, experience, sense-making and emergency.
\end{abstract}

Keywords: En-action. Human Motricity. Phenomenology.

\section{RESUMO}

O presente trabalho pretende uma análise de vinculação entre as perspectivas teóricas em-ação, motricidade e fenomenologia com o propósito de estabelecer articulações, tanto em nível conceitual como programático dos campos de estudo mencionados. Nesse sentido, assevera-se que desde a perspectiva de seus fundamentos se encontram a mesma raiz, e que as possibilidades de encontro são absolutamente complementares. A fenomenologia como ponto de partida do enfoque da em-ação da motricidade cria condições ótimas para a confluência e reciprocidade de métodos e categorias de análise da problemática existencial na atualidade. O núcleo ou eixo central do enfoque da em-ação é constituído pelos seguintes aspectos: autonomia, encarnação, experiência, fazer-sentido e emergência.

Palavras-chave: Em-ação. Motricidade Humana. Fenomenologia.

\footnotetext{
${ }^{1}$ Profesor de la Facultad de Filosofía y Humanidades de la UACh. E-mail: seatoro@gmail.com
} 


\section{Presentación}

Desde la fenomenología tradicional (HUSSERL, 2013; HEIDEGGER, 1997; MERLEAU-PONTY, 2000) la dimensión situada y encarnada de la existencia ha ido asumiendo un rol fundamental dentro de esta línea de pensamiento y desarrollo filosófico. Del mismo modo, una de sus expresiones con más desarrollo ha sido el término Motricidad, profundizado por Merleau-Ponty hasta la aproximación más condensada y compleja, a saber, intencionalidad operante. De igual forma y a la luz de estas bases se han propiciado programas de investigación científico, educativo, estético, psicológico y social. En este sentido, la neurofenomenología y su perspectiva en-activa (VARELA; THOMPSON; ROSCH, 2011; VARELA, 2016; NOË, 2004; THOMPSON, 2007; GALLAGHER; SCHMICKING, 2010; STEWARD; GAPENNE; DI PAOLO, 2010; GALLAGHER; ZAHAVI, 2014) como la Ciencia de la Motricidad Humana de Manuel Sérgio (SÉRGIO, 1987; TRIGO et al., 2010; TRIGO, 2015; TORO; VALENZUELA, 2012). Si bien se pueden estudiar por separado, inevitablemente se llegará a las otras de alguna u otra forma, más allá de las diferencias terminológicas y procedimentales, en el fondo de la cuestión se trata de una misma base y enfoque con diferencias de horizonte y punto de partida, pero desde una misma compresión y base epistémica.

La fenomenología como orientación y fundamento sobre la experiencia humana, su relación y co-constitución de sí mismo y del mundo, permiten que tanto el enfoque en-activo y la motricidad humana se encuentren en muchos aspectos, desde lo conceptual a lo metodológico.

El presente trabajo pretende iniciar esta discusión y explicitar los puntos de encuentro más evidentes. Hemos optado por ir describiendo la en-acción como sentido y programa investigativo en primer lugar, y posteriormente profundizar en algunos elementos de base de la motricidad humana desde la perspectiva de Manuel Sérgio. La fenomenología la trataremos dentro de las descripciones de ambas propuestas, pues como se ha dicho, está presente en las dos aproximaciones.

\section{LA EN-ACCIÓN: LA REPRESENTACIÓN FUERA DE LUGAR}

Cabe señalar que el origen de este término surge desde las ciencias cognitivas, las cuales se han desarrollado enormemente en los últimos 50 años. Sin duda es una enorme tarea reconocer y describir dicho desarrollo, esfuerzo por cierto que escapa a los intereses y contexto de este trabajo, sin embargo, cabe señalar brevemente que estos estuvieron impregnados de la famosa distinción entre mente y cuerpo, entre lo material e inmaterial, entre el software y el hardware, el procesamiento de la información o el conexionismo (VARELA; THOMPSON; ROSCH, 2011). En tales enfoques se buscaba y orientaba desde una comprensión ontológicamente dualista y escindida tanto del ser humano, como de su comportamiento.

Estas ideas siguieron la metáfora de la mente como un computador, el concepto de representación mental, la mente misma como el contenido del cerebro que funcionaba como una suerte de recipiente o contenedor. Estas perspectivas asumían una visión representacionista de la realidad (VARELA, 2005), acorde a la ontología cartesiana y su correspondiente epistemológico.

El problema era que estos planteamientos no daban cuenta, tanto neurológicamente como desde el punto de vista de la experiencia de la cotidianidad, el 
devenir humano y de la emergencia o surgimiento de lo que llamamos subjetividad, conciencia o mundo.

En ese contexto y desde una mirada profundamente fenomenológica, es decir, no fragmentaria, orientada hacia la experiencia de subjetividad, de conciencia y de mundo es que surge el enfoque en-activo o de en-acción.

La primera vez que se planteó la en-acción como programa investigativo fue en el año 1991 a través de Varela, Thompson y Rosch ${ }^{2}$. En dicho texto, los autores hacen mención que la denominación o creación del neologismo en-acción se produce en la raíz del verbo "to en-act", que expresa la idea de poner en acto, pero el sentido que más se acerca es hacer emerger o desplegar. Thompson (2007) profundiza su significado mencionando que se trata de un desempeño, de un hacer concreto en diferentes dimensiones o aspectos dependiendo del contexto y la repercusión sensorio-emocional que se genera en el actor. A su entender, el centro de este enfoque se entiende en la medida que comprendemos las características invariantes de los seres vivos desde su conformación y estructura molecular hasta sus expresiones más complejas como especie, tanto individual como social, que están en un acople estructural con su entorno. Desde ese acople y en virtud de sus condiciones y posibilidades de despliegue en su devenir, emerge lo que definimos como cognición y existencia al mismo tiempo.

En otras palabras y de manera sintética, este enfoque parte de los siguientes presupuestos, según Thompson (2007):

1. Los seres vivos son agentes autónomos que se originan y mantienen activamente desde la estructura y la dinámica que genera y lo genera [Autopoiesis de Maturana y Varela (1994)] $]^{3}$.

2. El sistema nervioso es un sistema dinámico que mantiene y genera sus patrones de coherencia y significado de actividad, conforme a superar como una red circular y recursiva de interacción neuronal. El sistema nervioso no procesa información en el sentido computacional, sino que crea significado.

3. La cognición es el ejercicio de "saber cómo" en una acción situada y encarnada las estructuras cognitivas emergen desde un acoplamiento recurrente de patrones de percepción y acción. El acoplamiento sensorio motor entre organismo y entorno modula, pero no determina, la formación de endógeno, dinámicos patrones de actividad neuronal que se presentan (informan) el acople sensorio motor.

4. El mundo de ser cognitivo no está preestablecido, una suerte de campo externo, representado internamente por su cerebro, sino, un dominio relacional en-actuado o construido por el agenciamiento de un ser autónomo y modelado de acoplamiento con el entorno. Si la experiencia no es un epifenómenal, sino central, para cualquier comprensión de la mente necesita ser investigada en un cuidadoso modo fenomenológico.

En este último aspecto aparecen dos elementos centrales dentro de esta aproximación. La primera se relaciona directamente con entender que la configuración

\footnotetext{
${ }^{2}$ Cabe señalar que esta fecha corresponde a la publicación del libro titulado "The embodied mind. Cognitive sciences and human experience". Su traducción al español fue el año 1997 y su título fue: "De cuerpo presente. Las ciencias cognitivas y la experiencia humana". Es importante señalar esta situación pues el presente trabajo se realizó a partir de la traducción en español.

${ }^{3}$ Este concepto ha sido muy convertido por tal motivo recurrimos a su versión original de los autores: "Una maquina autopoiética es una maquina organizada como un sistema de procesos de producción de componentes concatenados de tal manera que producen componentes que: - Genera los procesos (relaciones) de producción de los producen a través de sus continuas interacciones y transformaciones, y Constituyen a la maquina como una unidad en el espacio físico" (MATURANA; VARELA, 1994, p. 69). Para una profundización y actualización de la discusión sobre el tema se sugiere ver: RAZETO BARRY, P.; RAMOS JILIBERTO, R. Autopoiesis un concepto vivo. Santiago de Chile: IFICC, 2013.
} 
de mundo (lo que percibido), no es algo pasivo que opera como un centro de recopilación y organización de la información, sino más bien, la percepción que aparece como una condición agente, una cualidad que en sí misma que va orientando y agenciando el mundo.

Noë $(2004 ; 2012)$ define esta forma de entender la percepción como una acción cuyo sentido en sí mismo es agenciar el mundo, desde el despliegue y actuación del mismo. De manera que la percepción no es algo que ocurre o que se tiene, sino algo que se hace. Por consecuencia, tampoco podría estar ubicada en una determinada y definida entidad orgánica humana, sino en todo su proceder, en todas sus identidades, sean estas centrales o periféricas. Ubicadas a nivel del sistema nervioso central o en el muscular, óseo, digestivo, entre otros. Se pierde en esto el sentido fragmentario de la percepción o tributario de un órgano o subsistema en particular.

De manera que el foco de atención cambia sustancialmente pues ahora debiéramos centrarnos al menos paralelamente tanto en las estructuras susceptibles de ser medidas dentro de una experiencia o fenómeno, como al mismo tiempo en la experiencia o vivencia desde la perspectiva en primera persona.

En ese esfuerzo caben todas las miradas posibles en tanto ciencias o campos de estudio que favorezcan y permitan una mejor comprensión e interpretación de la experiencia, del fenómeno que emerge en el actuar de cada ser vivo y sobre todo en el caso de lo humano. Pues la experiencia aparece posterior a la acción, la significancia residual del sentido desplegado, de aquello que se vivenció tanto a nivel de concretar el acto desarrollado, como las diferentes modulaciones que como actor se presenta y produce en el mismo despliegue.

De esto último surge la segunda consecuencia de la percepción como acción. En el sentido de su posibilidad de estudio se presenta la fenomenología desde sus principios y herramientas de indagación que respeten y expliciten los procesos que configura la experiencia desde la persona misma que está en la situación (SCHMICKING, 2010; VAN MANEN, 2003). Puesto que en definitiva la conciencia siempre es sobre algo o desde algo, más allá del concurso de lo racional o lógico que se genera desde el lenguaje, cada ser vivo tiene de alguna manera un principio de unidad y autoreferencia, se constituye sobre sí mismo desde el flujo de su operacionar, al mismo tiempo que siente el mundo se siente a sí mismo, y desde la construcción de coordinaciones con otros seres vivos es que se genera la diferencia y conciencia sobre sí, de lo otro y de los semejantes o diferentes. Como diría Paulo Dantas la conciencia es una vivencia intencional (DANTAS, 2001).

Para que esta emergencia desde el actuar se haga más clara y pertinente, para desprender el sentido de la experiencia (MERLEAU-PONTY, 2006) es mucho más adecuado el concurso de diferentes descripciones científicas o de áreas de estudio que pueden favorecer la profundidad y ampliación de la existencia en despliegue.

Dada la complejidad y versatilidad de los sentidos y experiencia y el fenómeno en sí, principalmente, que los sentidos sobrepasan largamente los conocimientos establecidos o convencionalmente validados, siempre dependerá de sus contextos y entornos culturales y territoriales, del devenir cultural y las conversaciones que se mantienen en el curso de aquellos que se estudian. La fenomenología permite salvaguardar hasta el último momento la dignidad y el valor en sí de la experiencia como constitución de conocer y de mundo.

Por cierto, una vez que la ciencia pone su atención en la subjetividad y la conciencia, en la experiencia como es vivida, entonces no se puede hacer sin fenomenología. A su vez la fenomenología debe ser reconocida y cultivada 
como un partner indispensable de las ciencias experimentales de la mente y la vida (THOMPSON, 2007, p. 14, Mi traducción).

Pues en estas condiciones se asumen los supuestos mencionados más arriba que tienen las siguientes consecuencias:

- La mente, entendida como el relato del propio devenir en un contexto cultural situado y de sus eventuales perspectivas, no fabrica el mundo, lo constituye.

- En-acción se manifiesta en la autonomía, que a su vez es una característica fundamental de la vida biológica y existe una profunda continuidad de vida y mente. Y en términos de la fenomenología (MERLEAU-PONTY, 2000; 2006) la intencionalidad es una característica fundamental del cuerpo vivido.

- Al igual que la fenomenología desde el enfoque en-activo, la subjetividad y la conciencia tienen que ser explicadas en relación a la autonomía e intencionalidad de vida. En el amplio sentido de vida, que abarca el organismo en dos dimensiones: como cuerpo vivido subjetivo y al mismo tiempo como mundo de la vida.

En tal sentido, el enfoque en-activo o en-acción podría ser considerado o construido como un tipo de naturalismo no reductivo ni funcionalista. Centrado en el despliegue de lo humano en sus contingencias directas, que demandan un actuar pertinente para mantener la continuidad del flujo existencial dentro de las condiciones de autopoiesis molecular y de relación con otros seres vivos de la misma especie y de otras también. Como un continuo entre la vivir y la "mente", por consecuencia la ciencia que la investiga como un proceso de caracterización y explicitación de las fases o etapas de ese continuo.

Estas ideas o al menos algunas de ellas se pueden avizorar en algunos predecesores del enfoque en-activo como la teoría del equilibrio sensorio motriz como base de la cognición de Piaget y Brunner, en la fenomenología existencial (HEIDEGGER, 1997; MERLEAU-PONTY, 2000), la visiones sobre la experiencia de Dewey (2014), las perspectivas de Jonas (2000) y los aportes de von Foerster (1996), y naturalmente la biología del conocer original de Maturana y Varela (1994).

Pero el núcleo o eje central del enfoque en-activo se puede graficar en los siguientes aspectos:

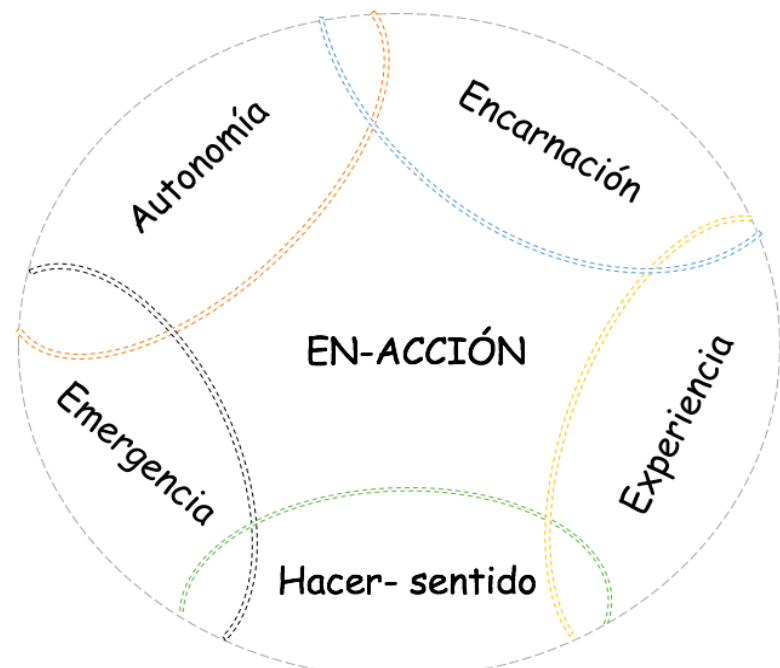

Figura 1: Núcleo o eje central del enfoque en-activo. 


\section{AUTONOMÍA, LOS ORGANISMOS VIVOS SON AUTÓNOMOS}

La autonomía como la libertad de auto-constitución o de constituirse así mismo, es decir, un sistema que su dinámica genera y sostiene una identidad.

Una identidad es generada cuando una red precaria de procesos dinámicos se transforma en una red cerrada operacionalmente, si por cualquier proceso dado $(\mathrm{P})$ que forme parte del sistema podemos encontrar entre sus condiciones habilitadoras otras que conforman el sistema; y podemos encontrar otro proceso en el mismo sistema que depende de P.

Esto que significa que en algún nivel de descripción, las condiciones que sostienen algún proceso en una red siempre incluye tales condiciones provistas por la operación de otros procesos en la red, y que los resultados de la actividad global es una unidad identificable en el mismo dominio o nivel de descripción (esto no significa, obviamente, que el sistema está aislado de interacciones con el entorno).

Autonomía como clausura operacional es intentar describir identidades generadoras de sí mismas en muchos niveles posibles, de manera que ellos no sólo responden a perturbaciones externas en el sentido tradicional de producir una acción apropiada para una determinada situación, ellos hacen un efecto activo y asimétricamente regulaciones sobre las condicionantes de intercambio con el entorno, y así continuamente, en-actúan un mundo o dominio cognitivo.

\section{ENCARNACIÓN}

La encarnación en el fondo nada más y nada menos es la comprensión sustantiva de un ser vivo, más que un cuerpo pasivo y contenedor, lo constitutivo de lo vivo es una red estructural que se genera y se siente a sí misma, que es capaz de percibir y percibirse. En general, los cuerpos no hacen o no manifiestan esta propiedad, por el contrario, se desgastan y dependen de factores externos para su generación, moldeamiento o desplazamiento al igual que las estructuras o sistemas autopoiéticos (producidos por agentes externos).

En tal sentido, la encarnación es más que las estructuras anatómicas o funcionales, puesto que son la expresión de desarrollo cultural que se manifiesta en hábitos y patrones de acción situados y desplegados concretamente. Así, los sentires o afecciones se vivencian y tienen lugar desde y en esa dimensión de lo vivo, específicamente o con mayor claridad en los mamíferos. Naturalmente se incluye lo que llamamos espíritu, pues en definitiva se entiende como la inspiración que se presenta en el desempeño de capacidades o habilidades más o menos valoradas dependiendo de la cultura en que nos encontremos. Es importante considerar que esta condición también tiene propiedades de adaptación sobre sí misma, como de ciertos aspectos externos para mantener su viabilidad existencial.

\section{EXPERIENCIA}

Como hemos dicho la experiencia constituye el elemento central para recoger y proyectar el modo de existencia y por consecuencia todos los dominios o dimensiones que posibilitan el mismo. En tal sentido, la comprensión de la experiencia como un proceso que recoge y perfila lo que constituye para el sujeto su vivencia, de esta forma el mundo se constituye desde el remante que permanece de lo experimentado. Esto convierte la experiencia en un eje central en todos los campos de actuación científica y 
profesional. Por ejemplo, dentro de la educación, el deporte, el arte o la vida cotidiana. En definitiva, la experiencia conforma lo que es otro componente del enfoque en-activo, el hacer sentido.

\section{HACER SENTIDO}

Se entiende desde el punto de partida que lo que se produce como cognición y comprensión de mundo no viene predado o es una representación de un mundo afuera del sujeto, objetivo y listo para ser adquirido, sino por el contrario, dicho mundo y la propia identidad se genera en y desde las relaciones que se establecen con lo que encuentre dentro del devenir, en resonancias o disonancias emocionales que dichos encuentros o relaciones hagan emerger. De manera que en la intensidad y profundidad de esos encuentros se van haciendo o constituyendo los sentidos sobre el mundo y sobre sí mismo (THOMPSON; DI PAOLO, 2014).

\section{EMERGENCIA}

Hace referencia a los procesos del conocer, y por tanto de aprender, y hacer sentido. Se van generando desde la emergencia tanto de situaciones y la consecuente demanda o despliegue de habilidades que den cuenta o permitan resolver la situación que emerge. Es cierto que en determinadas culturas se expresan y permanecen ciertos patrones de acción, y que la emergencia se da desde la recursividad de experiencias ya realizadas, pero dado la dinámica molecular que estructura a los seres vivos como también las contingencias a las cuales se ve enfrentado, favorecen y permiten que la estabilidad del sistema se genere en una emergencia permanente. Se puede apreciar en situaciones de juego o de danza, por ejemplo, en la interacción directa con semejantes o diferentes jugadores donde emerge la identidad e individualidad. Lo interesante, desde nuestro punto de vista es que dicha emergencia siempre será en relación. Por tanto, no se puede entender un ser vivo en sí mismo y para sí mismo sino constitutivo como emergentemente autónomo para la relación.

Parafraseando a Varela (2016), podemos decir, que lo encarnado, el cerebro no se pone en acción con todo lo que comprende el organismo para hacer funcionar la mente, más bien, es la emergencia de la acción emprendida, cuando se produce el diálogo, el lenguaje, que surge la mente. De igual forma, el fenómeno social emerge como producto de la interacción de los actores sociales, así emerge la mente también, es decir, desde la armonía de los distintos componentes del sistema nervioso. Desde el principio la sincronía o armonía oscilatoria que genera una experiencia denominada YO, que es siempre dinámica. Esta armonía va fluctuando y variando momento a momento (tiene una continuidad en la acción). Pero es discontinua como fenómeno neurológico.

Como hemos revisado todo el tiempo, hasta aquí hemos estado hablando de encarnación o dicho de otro modo de motricidad.

\section{MOTRICIDAD DESDE INTENCIONALIDAD OPERANTE A LA MILITANCIA}

González (2013) plantea que en los organismos multicelulares que tienen sistema nervioso, el desplazamiento, su acción, en definitiva, es parte constitutiva de su vida y de su experiencia. No se pueden comprender sin la acción, están constituidos para actuar y desenvolverse en su entorno y desde allí constituyen todo su conocer y vivir. Incluso antes de la imagen o de la idea, está la experiencia senso-emo-operacional. Al decir de Simondon (2013) no hay imagen, ni idea, menos un sueño o una creación sin 
motricidad. Así también, Merleau-Ponty (2000), nos dice que la motricidad es el sentido de todas las significaciones.

Ahora bien, ¿qué entendemos por motricidad?, otra vez mas allá de las definiciones debemos en primer lugar situarnos desde alguna matriz comprensiva.

Tal fue el caso de Manuel Sérgio $(1987 ; 1999 ; 2015)$ quién se plantea acerca de la motricidad desde la fenomenología como punto de partida. Iniciando la comprensión de motricidad como intencionalidad operante de Merleau-Ponty (2000), como la expresión del sentido y desde la definición del propio cuerpo, traducción de la categoría alemana Leib $^{4}$ referida por Husserl (2013).

Tal opción significó a la luz de su autor un corte epistemológico con la Educación Física heredera del cartesianismo francés y del empirismo inglés, que buscaba instalar una visión de lo humano coherente con una epistemología dualista, positivista, funcional y pro-capitalista. De manera que la Motricidad Humana desde Manuel Sérgio surge al mismo tiempo como un quiebre sobre el objeto de estudio como la forma en que este se aborda. Asumiendo toda la multi-dimensionalidad y complejidad, al mismo tiempo, que su condición de ser ético, social y político en todo lo que realiza en el existir humano.

Por tanto, la $\mathrm{CMH}$ denuncia, en la medicina y en la Educación Física, el uso y el abuso de la investigación cuantitativa y el desmedido desconocimiento de la investigación cualitativa. Y esto porque la epistemología de una ciencia del hombre tiene que asumir hasta las últimas consecuencias el carácter histórico-cultural de su objeto (en este caso, la motricidad humana) y del propio conocimiento como creación humana. La epistemología positivista siempre fue hostil a la introducción, en las ciencias, de la subjetividad, de la emoción, del deseo etc. Si todo lo real es complejo, lo cuantitativo y lo cualitativo deben integrarse en cualquier investigación científica.

Así asume toda la experiencia humana y todos sus campos de despliegue como eventuales y posibles de ser estudiados, pues la motricidad humana es en definitiva lo que permite existir y asumir un modo de existencia. Ya sea en el juego, la danza, el deporte, el teatro, la ergonomía o cualquier otra manifestación humana, pues en tanto humana es motricidad. Esto supera incluso las ideas más desarrolladas en esa época expresadas en las diferentes corrientes de la motricidad humana, por ejemplo, Livingston (1997) explicitaba que la motricidad humana tenía tres grandes funciones, a saber:

- Lo visceral, fundamentalmente entendido como los procesos y dinámicas internas propias de la organicidad que permiten los proceso bio-energéticos y funcionales en tanto estructura y organización fisio-termodinámica.

- La expresión de los propios estados de sentido y significancia en el contexto relacional en que se encuentra los organismos vivientes, específicamente en los mamíferos, con especial atención en la complejidad simbólica del existir humano. La expresión de emociones, sentimientos o ideas que se construyen y dan lugar en el devenir.

- El efectuar o hacer, es decir los despliegues de acción que permiten la presencia y actuación en los niveles relacionales en que se encuentra en ser humano en particular, que le permite mantenerse y relacionarse en los distintos dominios o campos de comportamiento, ya sea social, biológico, relacional o cultural.

\footnotetext{
${ }^{4}$ Cabe señalar que la palabra Leib tiene la misma raíz de leben (vida) y proximidad con liebe referida a amor. También que es diferente de Korper que refiere a cuerpo. Se ha asumido que Leib sería el cuerpo fenomenológico, es decir la experiencia de ser cuerpo, no de tener que es más cercana a la palabra Korper. En alemán antiguo la palabra $L \ddot{p} p$ se usaba tanto para vida como para estar presente o encarnado.
} 
Estos tres tipos de motricidad con Manuel Sérgio se amplían a las consideraciones de la dimensión política y trascendental de lo humano, vale decir, la superación de las limitaciones como un trascender a la dificultad de un estatus diferenciado de la motricidad, la única en un nivel ontológico no solo instrumental, aspecto propio de la fenomenología. De la misma manera, la dimensión política en el sentido que todo lo que se hace dentro de la actuación humana desde la libertad y relación social (ARENDT, 1993) es de suyo y propiamente política. Impronta una forma de entender y adherir a un tipo de organización social y un orden ético que lo orienta.

El planteamiento de este autor nos sitúa en un compromiso epistémico diferente del tradicional arraigado en la aparente neutralidad científica, más bien implica un compromiso y testimonio que en lo humano, en tanto ser emocional, sintiente, autopoiético y relacional, no puede desprenderse de sus orientaciones o prevalencias, al contrario, tenderá permanentemente a buscarlas y desarrollarlas en los diferentes campos de actuación que se encuentre.

En tal sentido la Motricidad Humana como ciencia o campo de estudio involucra el desarrollo humano en su más amplio sentido, que en la actualidad está íntimamente vinculado al desarrollo ecológico y planetario (DUSSEL, 2015; BOFF, 2016). Dado que la condición de ser vivo desde lo motricio o encarnado es en sí mismo un punto de vista, un lugar en sí mismo y para sí mismo que sólo se constituye en la relación con el entorno que lo hace posible ${ }^{5}$. De manera que el entendimiento de lo humano fuera de su contexto, natural y cultural, siempre será a lo menos parcial e inoportuno. No hay humanidad fuera de una biosfera, de allí que la motricidad implica lo que hacemos dentro de esta biosfera y para la biosfera. En tal sentido, la motricidad se amplía desde lo autónomo de lo individual hacia lo eco-relacional, la eco-motricidad. Pues somos constituyentes y producto de ese mismo ambiente, y lo que hacemos en tanto seres autónomos se hace dentro de esa condición de posibilidad, afectándola y modificándola. Tal modificación por una consecuencia obvia afectará directamente nuestro actuar. Es decir, metafóricamente hablando, caminamos nuestro propio sendero, somos camino y caminante al mismo tiempo, nos construimos desde y para el entorno.

De manera que la perspectiva de la motricidad implica, desde una visón actual o mejor dicho a la altura de lo conocimientos actuales sobre lo que llamamos realidad, su dimensión cuántica y sistémica, un abordaje y compromiso más profundo desde lo que hemos realizado históricamente, pero superándolo en términos de responsabilidad con la multidimensionalidad y contexto planetario. Pues ha sido la motricidad humana, desde una visión fragmentaria y dualista, que nos ha llevado a la actual condición social y planetaria. Con un conocimiento abrumador e histórico para la especie humana, pero al mismo tiempo en la situación más riesgosa para la vida en el planeta (BOFF, 2016; DUSSEL, 2015) en términos de marginalización, racismo y cambio climático.

En estos términos la motricidad humana no sólo se reduce a los procesos filo y ontogenéticos, sino también políticos y éticos. Donde todos los campos de estudio y las ciencias concurren para una mejor comprensión y desarrollo de modos de existencia que den cuenta tanto de las autonomías humanas, como de las relaciones que permiten una vida buena, en el sentido de las etnias originarias de América.

En síntesis, podemos atrevernos a realizar una definición de lo que hoy sería la motricidad humana, situados desde un continente con particularidades y situaciones

\footnotetext{
${ }^{5}$ Normalmente olvidamos lo fundamental que es lo que llamamos entorno o ambiente, solo permitamos recordar que el ser humano necesita llevarse el ambiente que le es propio, si desea entrar en contextos o entornos muy diferentes a la vida en la superficie del planeta, es lo que se hace cuando se viaja a la estratosfera o ambiente subacuáticos. Debemos llevar nuestro ambiente, en este sentido, más que ambiente es una piel que permite la existencia de la vida y por cierto la vida humana.
} 
diferenciadas de Europa o Norte América o mejor dicho Abya Yala ${ }^{6}$. Proponer entender la motricidad humana como el hacer-se mundo, toda vez que implica la autonomía y la relación al mismo tiempo, impronta que es una experiencia activa de doble dimensionalidad, lo propio y el contexto, lo individuado y colectivo, lo personal y comunitario. Al hacer voy y actúo el mundo, como también, me experimento y modulo en tanto autonomía en relación. Este aporte reconoce la complejidad de los fundamentos propuestos por Manuel Sérgio, pero al mismo tiempo se concretiza en un contexto latino y de doble dirección, como una condición de reciprocidad y convergencia dialógica con el entorno, una confluencia que se permiten mutuamente, no hay especie sin nicho, y el nicho de lo humano es la relación reciproca con su entorno y eso incluye lo biótico como lo no biótico.

\section{VÍNCULOS ENTRE EN-ACCIÓN Y MOTRICIDAD}

Los vínculos que se pueden establecer, a la luz de este autor, son muchos y significantes, más bien podríamos decir que son dos vertientes de un mismo afluente que se encuentran en las bases de comprensión y análisis, pero que se complementan desde dos campos de conocimientos diferentes, pero unidos por su horizonte de aportar y profundizar en la vida y desarrollo del vivir humano.

Ambos entienden que el saber en definitiva es un saber cómo, una acción, un proceder que tiene un carácter fundamental dentro de los procesos de los seres vivos. El cambio del conocer, si se produce desde el contenido, de lo que se ve por referencia, son cambios muy limitados y poco relevantes dentro del vivir. Pues lo que organiza el actuar está dado por el sentir del mundo, lo afectivo o emocional, dado que:

- Siempre hay un tono emocional y lo orgánico siempre aparece como pre constituido. El conocer es fundamentalmente un flujo, donde lo que predomina, la atención o la concentración está dado por el tono emocional que esa actividad demanda o dispone, por la postura o actitud, en otras palabras.

- La experiencia no está en la cabeza, sino en la periferia, esta descentralizada, es una confluencia de muchos componentes, lo emocional, los postural, lo relacional, el devenir histórico, entre otros aspectos.

- El saber es la manifestación o excedente de ese flujo.

- El aprendizaje, es precisamente un fenómeno o proceso que se da desde ahí, desde la resonancia emocional que permite la guía y constancia de un cambio de comportamiento en función de un mejor acople con el entorno.

- Estos procesos son pre-reflexivos, pre categoriales y están demandados por la contingencia, elemento primordial en la acción (HENRY, 2007). Estar consciente de dichos procesos involucra estar más presentes y más abiertos a la contingencia (VARELA; THOMPSON; ROSCH, 2011).

- Al examinar lo vivido, lo primero que aparece es el sistema motricio, es decir el despliegue-sentido, antes que la categoría, es lo que le da contenido a la percepción.

- Todas estas identidades son concurrentes, cuando mencionamos y aparece lo mencionado, pero no aparece descolgado, sino integrado, como una totalidad.

- El lenguaje y su unidad, la palabra están basadas, enraizadas en lo prelingüístico, la motricidad, en la coordinación o cohacer-se.

- Lo transitorio lo constituyente e inmanente, la limitud es radical para la conciencia de la transformación.

\footnotetext{
${ }^{6}$ Nombre asumido por los pueblos originarios en vez de América, de origen de la etnia Kuna de Panamá.
} 

relacionar.

- Así también la fonología, lo sonoro está encarnado, solo así se puede asimilar o

- El mundo no se viene encima, el vivir ya es una interpretación permanente.

- Somos tradición, especie antes que individuos, linajes, filogenia y la ontogenia.

- De manera que si llego a interpretar (acción) que el mundo no cambia es porque me estoy contando un cuento representacional, no depende de mí, no obstante nos hacemos mutuamente.

- La imaginación es lo más constitutivo de la vida humana, aquí emerge la creatividad.

- La emoción es lo más constitutivo de la razón, cada cosa que pasa surge a partir de una disposición emocional.

- Mi mente no existe sin tu mente, mi relato sobre mi mundo es una coconstitución.

- Lo que surge en tanto individuo sólo es posible en la resonancia, entrelazamiento con un prójimo.

Estos elementos son algunos que podemos extraer y explicitar de este pequeño ejercicio, sin duda podemos vislumbrar y constituir muchos más, pues en definitiva ambas perspectivas o programas de investigación, pretende devolver la responsabilidad del modo de existencia a los propios seres que crean interpretación y mundo.

La responsabilidad es entonces darnos cuenta que el modo de vida que llevamos es lo que hemos ido decidiendo y ejerciendo desde la tradición a la deliberación, por años y por consecuencia dependerá no sólo de los descubrimientos científicos, o mejor dicho nuestros descubrimientos científicos estarán dados y situados dentro de un marco ético y de opciones que dan sentido y explican nuestra situación, nuestra motricidad, nuestra en-acción.

En-actuar, hacer-se mundo, o constituir un modo de existencia que propicie mejores condiciones de vida de otros vivientes es una responsabilidad que no podemos eludir. Para transitar de una mismidad (sentimiento de lo propio) a una nosomidad o desde una auto-nomía a una eco-nomía. Es la problemática de nuestro tiempo, una motricidad así es lo que menos podemos aspirar.

\section{REFERENCIAS}

ARENDT, H. La condición humana. Barcelona: Paidos, 1993.

BOFF, L. La tierra está en nuestras manos. Una nueva visión del planeta y de la humanidad. Maliaño: Salterrae, 2016.

DANTAS, P. A intencionalidade do corpo próprio. Lisboa: Instituto Piaget, 2001.

DEWEY, J. Naturaleza humana y conducta. Introducción a la psicología social. Mexico DF: Fondo de la Cultura Económica, 2014.

DUSSEL, E. Filosofías del sur. Descolonización y transmodernidad. Mexico, DF: Akal, 2015.

GALLAGHER, S.; SCHMICKING, D. Handbook of phenomenology and cognitive sciences. Netherlands: Springers, 2010.

GALLAGHER, S.; ZAHAVI, D. La mente fenomenológica. 2. ed. Madrid: Alianza Editorial, 2014.

HEIDEGGER, M. Ser y tiempo. Santiago de Chile: Editorial Universitaria, 1997.

HENRY, M. Filosofía y fenomenología del cuerpo. Salamanca: Sigueme, 2007. 
Sergio Toro Arévalo

HUSSERL, E. Ideas relativas a una fenomenología pura ya una filosofía fenomenológica. México DF: Fondo de la Cultura Económica, 2013.

JONAS, H. Principio vida. Hacia una filosofía biológica. Madrid: Trotta, 2000.

LIVINGSTON, R. Desarrollo del cerebro humano. En: VARELA, F.; HAYWARD, J. Un puente para dos miradas. Conversaciones con el Dalai Lama sobre las ciencias de la mente. Santiago de Chile: Dolmen, 1997. p. 213-264.

MATURANA, F.; VARELA, F. De máquinas a seres vivos. Autopoiesis: La organización de lo viviente. 2. ed. Santiago de Chile: Editorial Universitaria, 1994.

MERLEAU-PONTY, M. Psicologia e pedagogia da criança. São Paulo: Martins Fontes, 2006.

MERLEAU-PONTY, M. Fenomenología de la percepción. Barcelona: Ed. Península, 2000.

NOË, A. La mente fuera de la cabeza. Madrid: Kairos, 2012.

NOË, A. Action in perception. Massachusetts: MIT Press, 2004.

SCHMICKING, D. A toolbox of phenomenological methods. En: GALLAGHER, S.; SCHMICKING, D. Handbook of phenomenology and cognitive science. Netherlands: Springer, 2010. p. 35-55.

SÉRGIO, M. O futebol e eu. Lisboa: Prime Books, 2015.

SÉRGIO, M. Um corte epistemológico. Da educação física à motricidade humana. Lisboa: Instituto Piaget, 1999.

SÉRGIO, M. Para uma epistemologia da motricidade humana. Lisboa: Compendium, 1987.

SIMONDON, G. Imaginación e invención. Buenos Aires: Cactus, 2013.

STEWARD, J.; GAPENNE, O.; DI PAOLO, E. Enaction. Toward a new paradigm for cognitive science. Massachusetts: MIT Press, 2010.

THOMPSON, E. Mind in life. Biology, phenomenology, and sciences of mind. Cambridge: Belknap Press of Harvard University Press, 2007.

THOMPSON, E.; DI PAOLO, E. The enective aproach. En: SHAPIRO, L. The Routledge handbook of embodied cognition. New York: Routledge, 2014. p. 68-78.

TORO, S., VALENZUELA, P. De la acción a la enacción, mas alla de la educación física y el movimiento. Estudios Pedagógicos, n. esp., 2012.

TRIGO, E. Manuel Sérgio un legado de su pensamiento. Pereira: Instituto Internacional del Saber, 2015.

TRIGO, E.; SÉRGIO, M.; GENÚ, M.; TORO, S. Retrospectivas de la motricidad humana. Pereira; Instituto Internacional del Saber, 2010.

VAN MANEN, M. Investigación educativa y experiencia vivida. Barcelona: Idea Books, 2003.

VARELA, F. El fenómeno de la vida. 2. ed. Santiago de Chile: Saez Editores, 2016.

VARELA, F. Conocer. Las ciencias cognitivas: tendencias y perspectivas. Cartografía de las ideas actuales. Barcelona: Gedisa, 2005.

VARELA, F.; THOMPSON, E.; ROSCH, E. De cuerpo presente. Las ciencias cognitivas y la experiencia humana. Barcelona: Gedisa, 2011. 
VON FOERSTER, H.; Semillas de la cibernética. Barcelona: Gedisa, 1996.

Recibido en: 17 oct. 2017.

Aprobado en: 30 oct. 2017. 